\title{
Beyond-All-Orders Instability in the Equatorial Kelvin Wave
}

\author{
Andrei Natarov \\ John P. Boyd \\ Department of Atmospheric, Oceanic \\ and Space Science and Laboratory for Scientific Computation, \\ University of Michigan, 2455 Hayward Avenue, Ann Arbor MI 48109 \\ jpboyd@engin.umich.edu; \\ http://www.engin.umich.edu:/ jpboyd/
}

August 24, 2000

Dynamics of Atmospheres and Oceans, in press. 


\begin{abstract}
The equatorial Kelvin wave is destabilized by cross-equatorial shear in a rather bizarre fashion. There is no well-defined inviscid neutral curve. Instead, using an exemplar the linear shear $U(y)=\epsilon y$ where $U$ is the mean zonal flow and $y$ is nondimensional latitude, the Kelvin wave is unstable for all $\epsilon$, however, small. We show through multiple precision numerical calculations that the imaginary part of the phase speed is proportional to the square of the unperturbed eigenfunction at the critical latitude, that is, $\Im(c) \propto \exp \left(-1 / \epsilon^{2}\right)$ for small $\epsilon$. Such exponential dependence on a small parameter implies that result lies "beyond-all-orders" in a perturbation series in powers of $\epsilon$. We connect our numerical work here with our previous study of a model problem through "beyond-all-orders" perturbation theory to argue that the Kelvin instability is a critical latitude phenomenon.
\end{abstract}

\title{
1 Introduction
}

The equatorially-trapped Kelvin wave is a dominant wave mode in the tropical ocean and atmosphere (Andrews, Holton and Leovy, 1987, Gill, 1982, Lindzen, 1990, and Philander, 1990). Therefore, it is important the destabilization of these waves by the strong zonal mean jets that are present in both the sea and the middle atmosphere. Previous studies of instabilities of equatorial waves include Ripa (1983), Dunkerton (1983), Stevens (1983), Ripa and Marinone (1983), Marinone and Ripa(1984), Boyd and Christidis (1982, 1983), Proehl(1996, 1998).

So-called "pancake instabilities" in the middle atmosphere (Hitchman et $a l ., 1988)$ have been identified as Kelvin waves destabilized by cross-equatorial variations in the mean zonal wind (Boyd and Christidis, 1982, 1983). Similarly, inertial instability, which is connected with unstable Kelvin waves, has been invoked as a mechanism for reducing equatorial asymetry in the mean zonal wind in the troposphere and equatorial ocean (Stevens, 1983, and Dunkerton, 1983). The relevance of Kelvin instabilities to the ocean is not known, but one cannot obviously answer this question until one actually has a theory for unstable Kelvin modes.

One remarkable finding of (Boyd and Christidis, 1982, 1983) is that there was no well-defined neutral point. For inviscid instabilities, the usual situation is illustrated by the left panel in Fig. 1. At finite value of the parameter $\epsilon_{c}$, two roots merge and form a complex conjugate pair for $\epsilon>\epsilon_{c}$. One can show that the imaginary part of the phase speed has an infinite slope with respect to the parameter at the neutral point $\epsilon=\epsilon_{c}$.

In contrast, the Kelvin wave seems to be unstable for all non-zero shears, however weak. The slope $d \Im / d \epsilon$ is not infinite but zero at $\epsilon=\epsilon_{c}=0$. It is easy to calculate a perturbation series in powers of the shear strength $\epsilon$ (Boyd, $1978 \mathrm{~d}$, e). However, this is real-valued to all orders; the instability is missed completely by the power series in $\epsilon$. The numerical computations suggest that 
the imaginary part of the eigenvalue is an exponential function of $1 / \epsilon$, and therefore lies "beyond all orders" in $\epsilon$.

Fig. 2 shows why this exponential dependence is plausible, using the particular case of a linear shear. The unperturbed zonal velocity and pressure fields of a Kelvin wave are proportional to $\exp \left(-(1 / 2) y^{2}\right)$ where $y$ is the nondimensional latitudinal coordinate defined in the next section and the nondimensional phase speed of the Kelvin wave is $c=1$. When the mean zonal current is $U(y)=\epsilon y$, the critical latitude where $c=U(y)$ and the linearized shallow water equations are singular is located at $y=y_{\text {critical }}=1 / \epsilon$. The amplitude of the unperturbed Kelvin wave pressure and zonal velocity is $\exp \left(-(1 / 2) / \epsilon^{2}\right)$ at the critical latitude, a consequence of (i) the very rapid decay of the eigenfunction with distance from the equator and (ii) the inverse relationship between the distance of the critical latitude from the equator and the shear strength $\epsilon$.

\section{Typical}

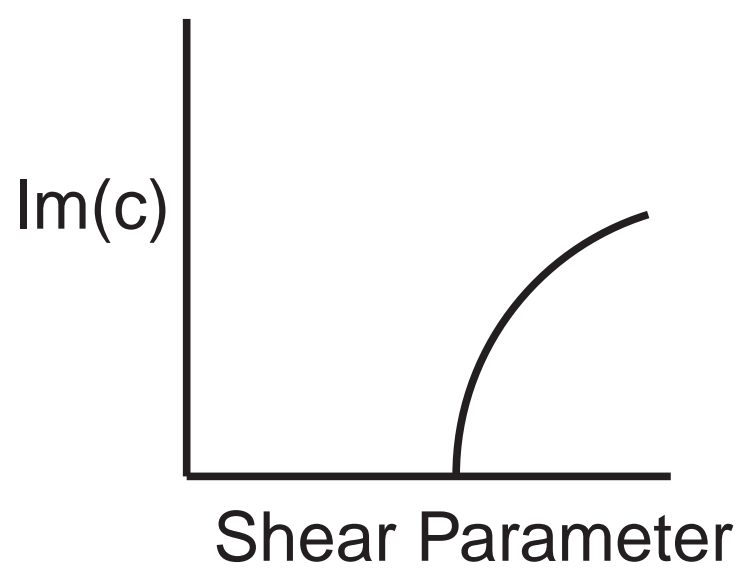

Kelvin

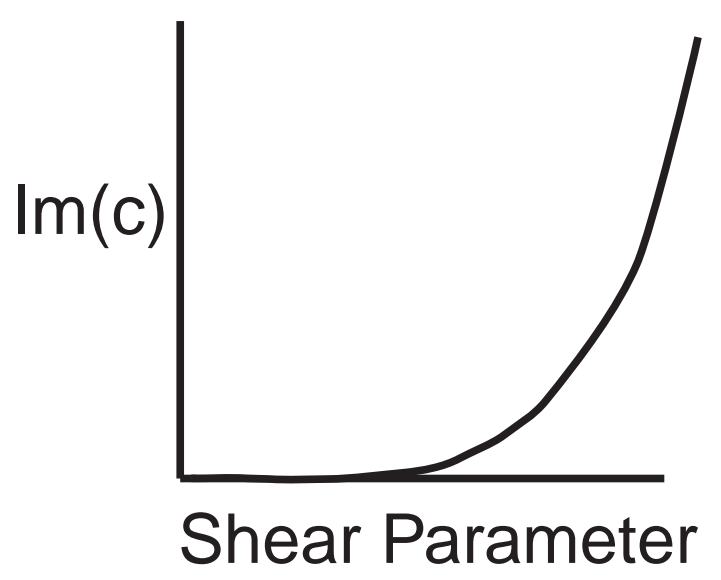

Figure 1: Left: $\Im(c)$ has a square-root singularity at some finite value of the shear parameter where $d \Im / d \epsilon=\infty$. Right: the Kelvin wave is unstable for all $\epsilon$ with an exponential growth in $\Im(c)$.

If the instability is a critical latitude effect, as conjectured by Boyd and Christidis $(1982,1983)$, then it is plausible that the growth rate of the instability would be proportional to the magnitude of the unperturbed eigenfunction at the critical latitude, at least in order of magnitude. Because this amplitude is $\exp \left(-(1 / 2) / \epsilon^{2}\right)$, it is reasonable that $\Im(\lambda)$ would be exponentially small in $1 / \epsilon$ as observed.

However, the numerical calculations, limited by the arithmurgical technology of 1982, were unable to accurately determine the form of the exponential. (A numerical curve-fit to an exponential in the Boyd-Christidis paper is misleading and inaccurate when extended to small $\epsilon$, as we show below.) How can 
one understand this apparent exponential dependence of growth rate on shear strength when this is invisible to ordinary perturbation theory?

In the last decade, there has been an explosion of interests in what is variously called "exponential asympotics" or "beyond-all-orders" perturbation theory. This is not a single method, but rather is a collection of half a dozen different strategies for calculating effects which are exponentially small in the reciprocal of the perturbation parameter as explain in the collection (Segur, Tanveer and Levine, 1991), the monographs(Boyd, 1998, and Jones, 1997), the long review (Boyd, 1999) and the mini-reviews (Berry and Howls, 1993, and Paris and Wood, 1995) .

This moved us to reexamine the Kelvin instability using the new tools of exponential asymptotics. Unfortunately, a direct assault on the shallow water wave equations is still rather formidable: a system of three equation in three unknowns. In the spirit of the oft-quoted witticism of Gene Golub - "an applied mathematician is one who solves almost the problem you ask him to" - we began our reexamination by solving a simple problem: the lowest mode ("ground state") of the "Hermite-with-pole" equation:

$$
u_{y y}+\left\{\epsilon \frac{1}{1+\epsilon y}-y^{2}\right\} u=\lambda u
$$

The unperturbed $(\epsilon=0)$ eigenfunction has the same shape as the Kelvin wave: $u(y ; \epsilon=0)=\exp \left(-(1 / 2) y^{2}\right)$. The perturbed eigenfunction has a logarithmic singularity at the "critical latitude" where the coefficient of the undifferentiated term is infinite; the branch cut is in the upper half-plane for reasons explained in Dickinson(1968).

In Boyd and Natarov(1998), we found that the imaginary part of the eigenvalue $\lambda$ is indeed an exponentially small function of the shear parameter $\epsilon$. However, there was a surprise: $\Im(\lambda)$ is not proportional to the amplitude of the unperturbed eigenfunction at the critical latitude, $\exp \left(-(1 / 2) / \epsilon^{2}\right)$ as expected, but rather to the SQUARE of the amplitude of the eigenfunction at $y=y_{\text {critical }}=-1 / \epsilon$ :

$$
\Im(\lambda)=\sqrt{\pi} \exp \left(-\frac{1}{\epsilon^{2}}\right)\left\{1-2 \epsilon \log \epsilon+(\log 2+\gamma) \epsilon+O\left(\log ^{2}(\epsilon) \epsilon^{2}\right)\right\}
$$

Is the same true of the $\mathrm{K}$ elvin wave? In this second part of a three-part study, we show through numerical calculations that it. The third, as yet unaccomplished part, is to extend exponential asymptotics to the shallow water wave equations on the equatorial beta-plane to derive an analytical approximation for $\Im(\lambda)$ as we have already done for the Hermite-with-pole equation. 

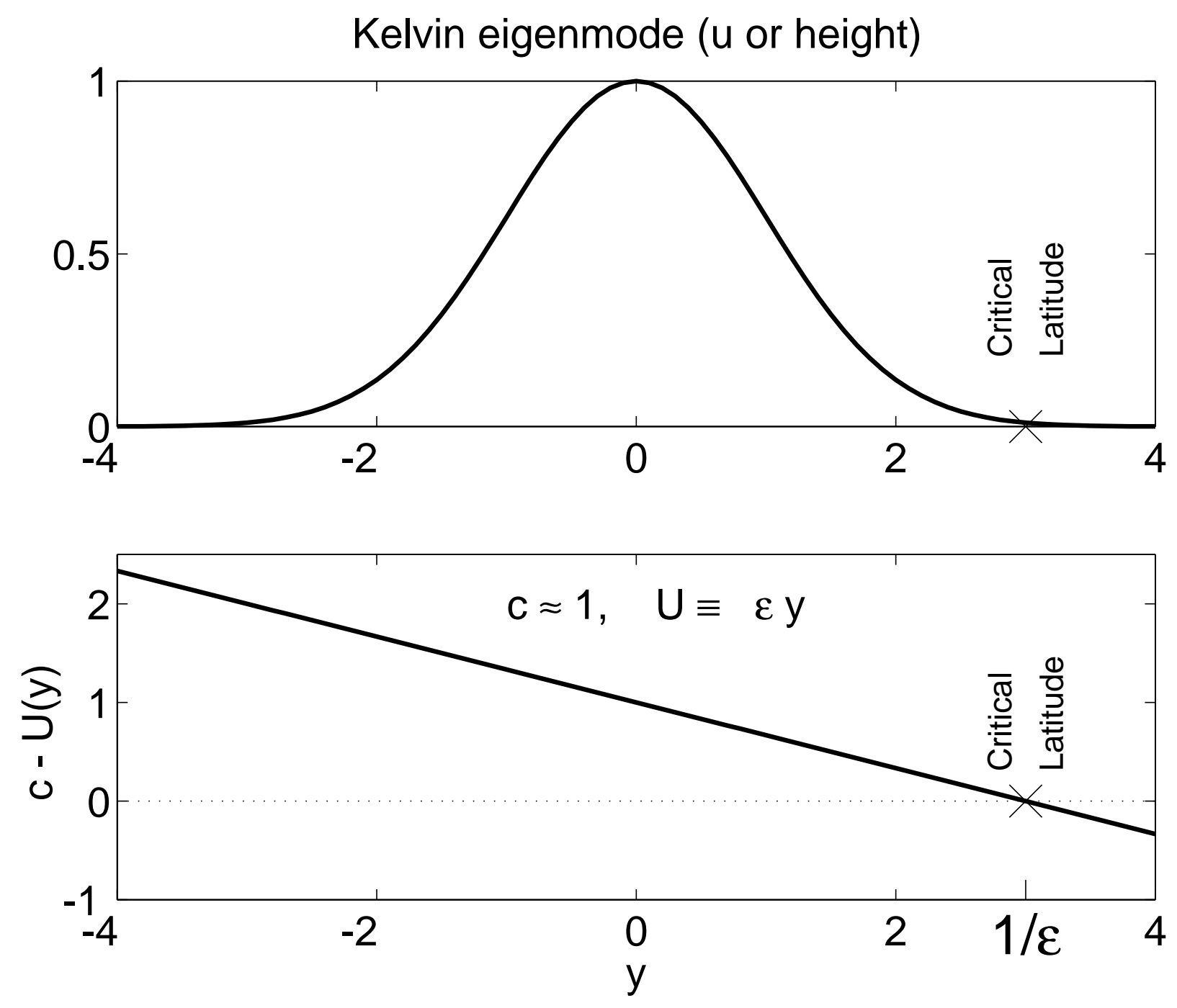

Figure 2: Top: schematic of a linearized Kelvin wave in a mean shear flow. Bottom: graph of $c-U(y)$ for the Kelvin wave for Couette flow, $U(y) \equiv \epsilon y$. The wave equation is singular, and the eigenmode has a critical latitude, at $y=-1 / \epsilon$ where $c-U$ is zero. The critical latitude creates an imaginary part in the phase speed $c$. However, $\Im(c)$ is exponentially small in $1 / \epsilon^{2}$ because the amplitude of the eigenfunction at $y=1 / \epsilon$ is $O\left(\exp \left(-1 / \epsilon^{2}\right)\right)$. 


\section{Numerical Solutions}

The shallow water equations in a moving reference frame are

$$
\begin{gathered}
(u-c) u_{x}+v u_{y}-y v+h_{x}=0 \\
(u-c) v_{x}+v v_{y}+y u+h_{y}=0 \\
\{(u-c) h\}_{x}+\{v h\}_{y}=0
\end{gathered}
$$

where $c$ is the phase speed and $h=1+\phi$ is the total depth. Linearization about the basic state $\{u, v, \phi\}=\{U(y), 0, \Phi(y)\}$ gives

$$
\begin{gathered}
(U-c) u_{x}+v U_{y}-y v+h_{x}=0 \\
(U-c) v_{x}+y u+h_{y}=0 \\
(U-c) h_{x}+\{1+\Phi\} u_{x}+\{1+\Phi\} v_{y}+\Phi_{y} v=0
\end{gathered}
$$

where the basic state is assumed to be geostrophically balanced.

When the shallow water is derived by separating variables in the equations of motion for a three-dimensional, compressible atmosphere, the result is identical to the system except that in the continuously stratified case, $\Phi(y) \equiv 0$ as explained in Boyd and Christidis $(1982,1983)$. (Of course, the interpretation of the shallow water wave equations is changed, too: the three-dimensional wave mode is the product of factors that depend only on $z$ times the solution of the shallow water set, which describes the latitude and longitude dependence of the wave.) For consistency with the earlier work, this is the set of equations we shall solve below except for the computations of Table 3 where we compare $\Phi=0$ with nonzero $\Phi$.

Because the primary purpose of this work is to study critical latitude effects in isolation from other possible forms of instability, we choose linear shear flow $U(y)=\epsilon y$ as basic state for further studies. Such profile does not have singularities in the complex $y$-plane, which could complicate the numerics, or inflection points. The only problem is that physically it cannot be supported by any geostrophic $\Phi(y)$, because the streamlines would hit the surface $\left(\Phi\left(y_{\text {surf }}\right)=-1\right)$ long before they reach critical latitude. This is a second reason to emphasize the atmospheric model, $\Phi \equiv 0$.

The search for normal mode solutions leads to a linear eigenvalue problem for $c$

$$
\left[\begin{array}{ccc}
i k U & -y+\partial_{y} U & i k \\
y & i k U & \partial_{y} \\
i k & \partial_{y} & i k U
\end{array}\right]\left[\begin{array}{l}
u \\
v \\
\phi
\end{array}\right]=i k c\left[\begin{array}{l}
u \\
v \\
\phi
\end{array}\right]
$$


We solve the above system numerically for $U(y)=\epsilon y$ using two different numerical algorithms. Because computational methods are very unhappy with poles and branch points on the integration domain, we avoided disaster at the critical latitude by deforming the contour of integration into the complexplane(Boyd, 1985; Boyd, 2000). Provided that the new and old contours are not separated by a singularity of the solution, the eigenvalue will be unchanged by the change in the integration interval.

Our first algorithm applied a Chebyshev pseudospectral discretization of the differential equation(Boyd, 1985; Boyd, 2000) with the complex mapping

$$
y=z-i \delta
$$

where $\delta$ is a positive constant. (The sign of $\delta$ implicitly forces the branch cut from the critical latitude singularity to lie in the correct half-plane.) Unfortunately, the Chebyshev method generates dense matrices; when the QR/QZ algorithm is employed to solve the matrix eigenproblem, the cost grows as the cube of $N$, the number of Chebyshev polynomials. The Chebyshev/QR scheme provided a useful independent verification of the shooting algorithm described below, but became too expensive for $\epsilon<0.3$. Note that as $\epsilon$ becomes smaller and $\Im(c)$, we need more and more basis functions or grid points so that the error is smaller than $\Im(c)$. Eventually $\Im(c)$ becomes small than the roundoff error and we have to switch to multiple precision shooting code implemented in MAPLE.

To solve the system using a "shooting" method(Ascher et al., 1995), it is convenient to reduce the system of three ordinary differential equations to two by eliminating the east-west velocity $u$ :

$$
\begin{aligned}
v_{y} & =F(v, \phi) \\
\phi_{y} & =G(v, \phi)
\end{aligned}
$$

where

$$
\begin{gathered}
F(v, \phi)=-\frac{y-U_{y}}{U-c} v+i k\left\{\frac{1}{U-c}-(U-c)\right\} \phi \\
G(v, \phi)=-\left\{\frac{y\left(y-U_{y}\right)}{i k(U-c)}+i k(U-c)\right\} v+\frac{y}{U-c} \phi
\end{gathered}
$$

supplemented with boundary conditions

$$
\phi, v \rightarrow 0 \text { as }|y| \rightarrow \infty
$$

which we solve by shooting from $-\infty$ and $+\infty$ (replaced by $-L$ and $L+1 / \epsilon$ in the actual implementation) toward the equator and matching the solution vectors at the equator. Marching was implemented using constant step RK4 routine. Secant method was used for matching, which appeared to be more 
Table 1: $k=0.8$

\begin{tabular}{|c|c|c|c|l|}
\hline$\epsilon$ & $\mathrm{N}$ & \multicolumn{1}{c}{ Digits } & $\Re(c)$ & $\Im(c)$ \\
\hline 0.90 & 200 & 20 & 1.02313038 & 0.019049736 \\
\hline 0.80 & 200 & 20 & 1.03655269 & 0.009866228 \\
\hline 0.70 & 200 & 20 & 1.04150483 & 0.004129622 \\
\hline 0.60 & 200 & 20 & 1.03979475 & 0.001247925 \\
\hline 0.55 & 200 & 40 & 1.03688905 & 0.000567376 \\
\hline 0.50 & 800 & 40 & 1.03315626 & 0.000213592 \\
\hline 0.40 & 800 & 40 & 1.02408866 & 0.000011666 \\
\hline 0.35 & 800 & 40 & 1.01932224 & $0.11645 \mathrm{e}-5$ \\
\hline 0.30 & 800 & 40 & 1.01474152 & $0.3889 \mathrm{e}-7$ \\
\hline 0.25 & 800 & 50 & 1.01055164 & $0.1709 \mathrm{e}-9$ \\
\hline 0.20 & 4000 & 50 & 1.00691550 & $0.1097 \mathrm{e}-13$ \\
\hline 0.19 & 4000 & 50 & 1.00626649 & $0.6317 \mathrm{e}-15$ \\
\hline 0.18 & 4000 & 50 & 1.00564567 & $0.2245 \mathrm{e}-16$ \\
\hline 0.17 & 4000 & 50 & 1.00505039 & $0.4044 \mathrm{e}-18$ \\
\hline
\end{tabular}

efficient than Newton's method. Typically it takes less than 10 iterations to converge.

The functions $F(v, \phi)$ and $G(v, \phi)$ on the right hand side of the above system are singular at the critical latitude. To avoid singularities the contour of integration dips just below the real axis near the critical latitude

$$
y=z-i \delta e^{-z^{2} / \sigma^{2}}
$$

where $\delta$ and $\sigma$, after some experimentation, were taken equal to 1. For large $\epsilon$, where 20 digits and 200 grid points sufficed, only three minutes of CPU time was needed. Our most time-consuming runs used 50-digit precision and 4000 grid points in latitude, requiring about 12 hours on an HP 9000 model $715 / 64$ workstation. The smallest linear shear for which we were able to obtain trustworthy results was $\epsilon=0.17$.

For smaller values one needs to use more digits and more points in the domain. Calculations with 100 digits and 4000 points for $\epsilon=0.15$ were inaccurate for $\Im(c)$, and going to more digits required such long run times that the network actually crashed before the job was done.

It turns out that the results are not sensitive to zonal wave number $k$ as illustrated in Fig. 3. For this reason, we have tabulated the real and imaginary parts of the eigenvalue and also graphed $\Im(c)$ versus $1 / \epsilon^{2}$ only for the representative but somewhat arbitrary case of $k=0.8$. (Table $1 ; N$ is the total number of grid points in latitude.)

Just as for the Hermite-with-Pole equation, the imaginary part of the phase speed of the Kelvin wave is proportional to the square of the amplitude of the unperturbed eigenfunction or in other words

$$
\Im(c) \sim \text { constant } \exp \left(-1 / \epsilon^{2}\right)
$$




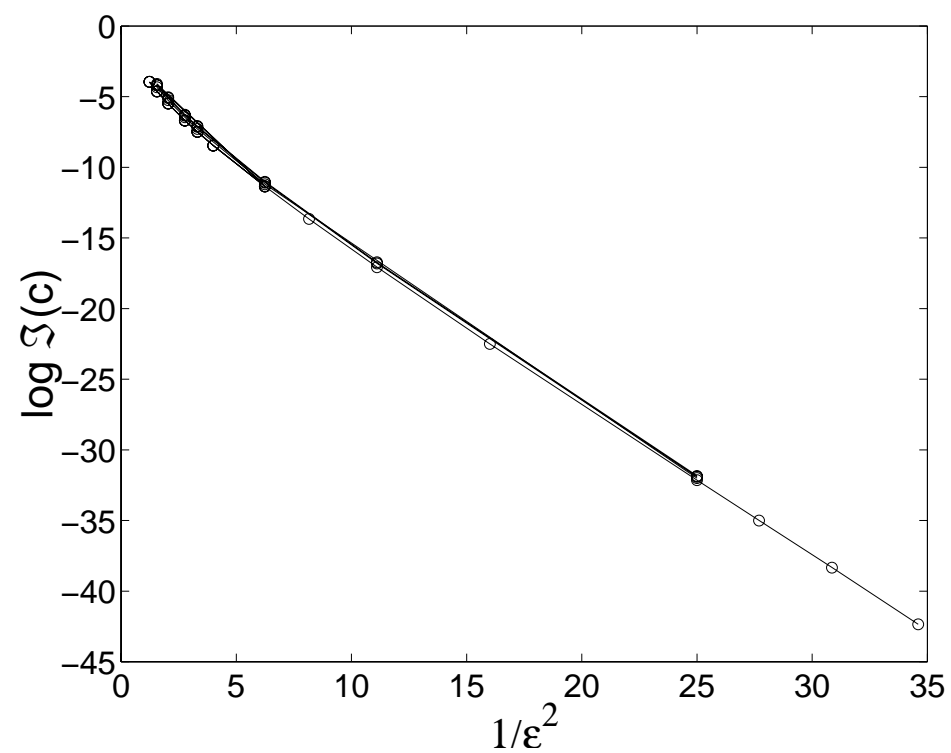

Figure 3: Composite graph for $\mathrm{k}=0.2,0.4,0.6,0.8$. $\Im(c) \sim \exp \left(-\frac{1}{\epsilon^{2}}\right)$ as $\epsilon \rightarrow 0$ and is not very sensitive to $k$.

Table 2: No critical latitude $k=0.8$

\begin{tabular}{|c|c|c|c|}
\hline$\epsilon$ & $\mathrm{N}$ & $\Re(c)$ & $\Im(c)$ \\
\hline 0.30 & 800 & 1.01473769 & $0.9 \mathrm{e}-42$ \\
\hline 0.25 & 800 & 1.01055153 & $0.4 \mathrm{e}-35$ \\
\hline 0.20 & 800 & 1.00691550 & $0.1 \mathrm{e}-45$ \\
\hline
\end{tabular}

This is illustrated in Fig. 4 where the numerical curve (solid-with-circles) is clearly asymptoting to the dashed line, which is Eq. 16.

This supports our conjecture that the instability is a critical latitude effect. To provide further evidence, we made calculations for a second mean flow profile. This is identical with the linear profile around the equator, but is constant for large $|y|$ and thus lacks a critical latitude:

$$
U(y)=\left\{\begin{array}{ccc}
-\mathcal{U} & \text { if } & y<-\mathcal{U} / \epsilon \\
\epsilon y & \text { if } & |y|<\mathcal{U} / \epsilon \\
\mathcal{U} & \text { if } & y>\mathcal{U} / \epsilon
\end{array}\right.
$$

where $\mathcal{U}=0.8$ (far enough from both critical latitude and equator).

The real part of the eigenvalue remains almost unaltered, while $\Im(c)$ drops by many orders of magnitude to roughly the roundoff error. This indicates that the existence of the critical latitude is indeed crucial for instability. 


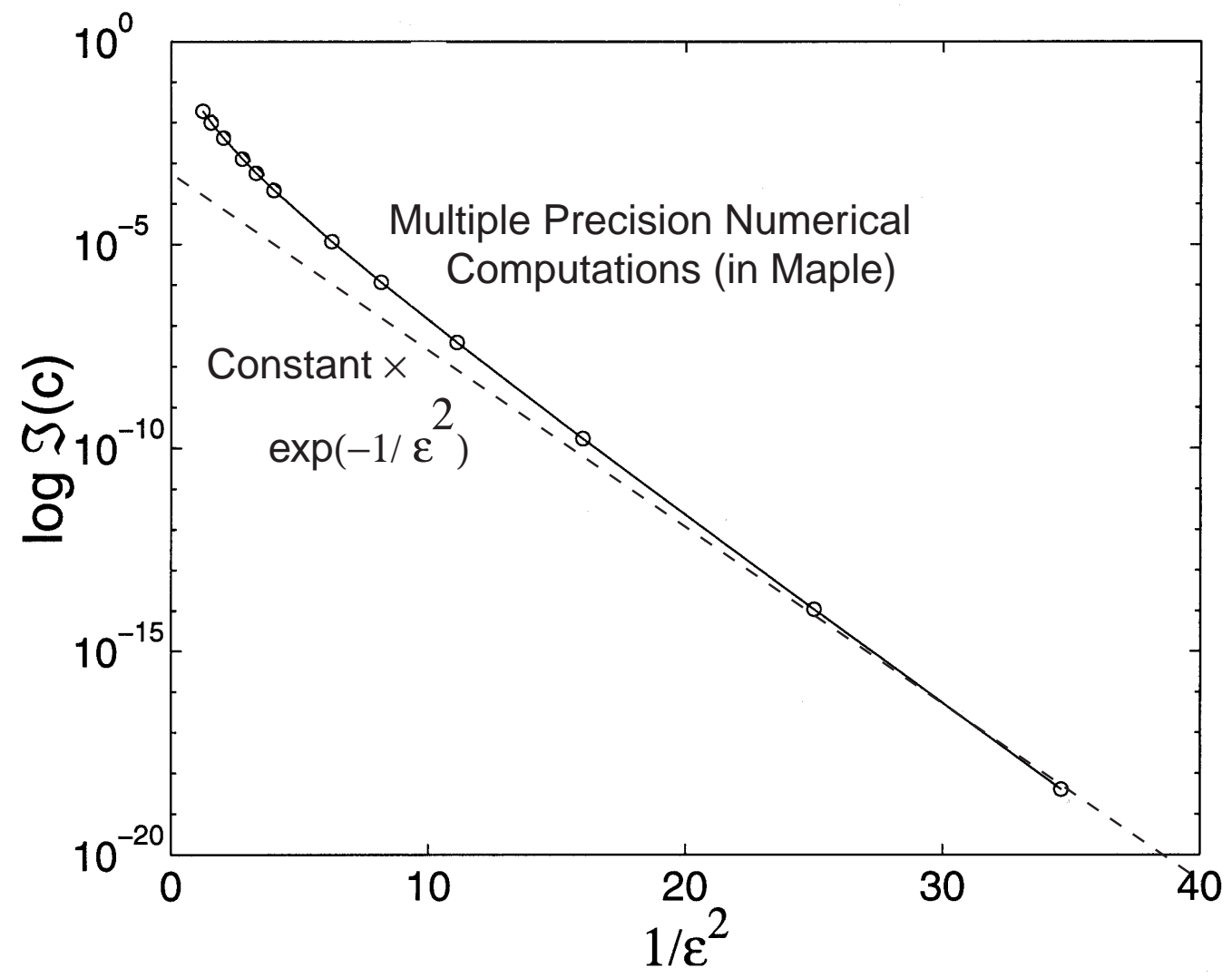

Figure 4: Solid-with-circles: Numerical calculations for $\Im(c)$ for $k=0.8$. Dashed: constant $\exp \left(-1 / \epsilon^{2}\right)$. 
Table 3: Off-equatorial jet results. For all experiments $w=0.1, N=1600, \delta=$ $0.4, \sigma=1.0$.

\begin{tabular}{|cc|cc|cl|}
\multicolumn{1}{r}{$U_{0}$} & $\epsilon$ & $\Re(c)$ & $\Im(c)$ & $\Re(c)[\Phi=0]$ & $\Im(c)[\Phi=0]$ \\
\hline 0.9 & 0.5 & 1.00460854 & .00000000 & 1.00508879 & .00000000 \\
1.1 & 0.5 & 1.00572669 & .00078746 & 1.00617015 & .00077398 \\
1.2 & 0.5 & 1.00404085 & .00373990 & 1.00508687 & .00398477 \\
1.3 & 0.5 & 1.00147460 & .00642911 & 1.00223319 & .00596192 \\
\hline 0.9 & 0.4 & 1.00047156 & .00000000 & $1.0005399 ?$ & .00000000 \\
1.1 & 0.4 & 1.00058580 & .00008100 & 1.00064961 & .00008306 \\
1.2 & 0.4 & 1.00042015 & $.0003374 ?$ & 1.00048877 & .00032438 \\
\hline
\end{tabular}

We also experimented with off-equatorial jets of the form

$$
\begin{gathered}
U(y)=U_{0} \exp \left(-\frac{(y-1 / \epsilon)^{2}}{w^{2}}\right) \\
\Phi(y)=-\int_{-\infty}^{y} \xi U(\xi) d \xi
\end{gathered}
$$

Because the phase speed of the equatorial Kelvin wave is always almost one, there will be a critical latitude only if $U_{0}>1$, and it is easy to check if instability is a critical latitude effect in such setting. The results summarized in Tab.3 confirm that instability only exists when $U_{0}>1$.

Next we make runs for $\Phi(y)$ set equal to zero, as in the Boyd-Christidis artilce. Tab. 3 shows that the results are very close to those produced in the full model runs.

\section{Mean Height Variations}

What happens when the shallow water wave equations include mean height variations, as is appropriate when using this system as a "one-and-a-half-layer" model of the tropical ocean? For a linear shear, the answer is that $\Phi\left(y_{s}\right)=0$ for some $y_{s}$ which is closer to the equator than the critical latitude so that there in fact is no critical latitude - and no Kelvin wave instability either.

For an off-equatorial jet, however, Kelvin wave instability is possible both with and without inclusion of the mean height field $\Phi(y)$. Table 3 shows that in this case, there are only slight differences between $\Phi=0$, i. e., the model solved in the rest of this article and in Boyd and Christidis (1982), and calculations with a mean height field $\Phi(y)$.

\section{Summary}

In this work, we performed numerical calculations to show that the growth rate for the Kelvin wave in weak shear behaves identically with the imaginary part 
of the eigenvalue for the Hermite-with-pole equation, studied through matched asymptotic expansions in our earlier article. This similarity suggests that our conjecture is correct: the instability is a critical latitude effect, at least for small $\epsilon$, and the growth rate is an exponential function of $1 / \epsilon^{2}$ because the eigenfunction is exponentially small at the far-from-the-equator critical latitude. To confirm this, we also examined a modified profile which was similar to the first near the equator, but was modified at high latitudes to remove the critical latitude. The real part of the phase speed is little altered by our mean flow surgery, but the imaginary part vanished to the limit of our multiple precision computations.

The unsolved problem is to understand the mechanics of the instability. Why is a critical latitude always destabilizing for a Kelvin wave, but not for a Rossby or gravity mode? How efficient is an off-equatorial critical latitude at adjusting the mean flow to a stable profile when the bulk of the Kelvin mode is still centered on the equator? Metaphorically, we have identified the scene of the crime (i. e., the critical latitude), but an in-depth understanding of precisely what happens at the critical latitude and how the mean flow adjusts are still not well understood.

\section{Acknowledgments}

This work was supported by the National Science Foundation through grant OCE9521133.

\section{References}

Andrews, D. G., J. R. Holton, and C. B. Leovy, 1987: Middle Atmosphere Dynamics. Boston: Academic Press.

Ascher, U. M., R. M. M. Mattheij, and R. D. Russell, 1995: Numerical Solution of Boundary Value Problems for Ordinary Differential Equations, volume 13 of Classics in Applied Mathematics. Philadelphia: Society for Industrial and Applied Mathematics (SIAM).

Berry, M. V. and C. J. Howls, 1993: Infinity interpreted Physics World, (pp. 35-39).

Boyd, J. P., 1978a: The effects of latitudinal shear on equatorial waves, Part I: Theory and methods Journal of the Atmospheric Sciences, 35, 2236-2258.

Boyd, J. P., 1978b: The effects of latitudinal shear on equatorial waves, Part II: Applications to the atmosphere Journal of the Atmospheric Sciences, 35, 2259-2267.

Boyd, J. P., 1985: Complex coordinate methods for hydrodynamic instabilities and Sturm-Liouville problems with an interior singularity Journal of Computational Physics, 57, 454-471. 
Boyd, J. P., 1998: Weakly Nonlocal Solitary Waves and Beyond-All-Orders Asymptotics: Generalized Solitons and Hyperasymptotic Perturbation Theory, volume 442 of Mathematics and Its Applications. Amsterdam: Kluwer $608 \mathrm{pp}$.

Boyd, J. P., 1999: The Devil's Invention: Asymptotics, superasymptotics and hyperasymptotics Acta Applicandae, 56(1), 1-98.

Boyd, J. P., 2000: Chebyshev and Fourier Spectral Methods. New York: Dover Second edition.

Boyd, J. P. and Z. D. Christidis, 1982: Low wavenumber instability on the equatorial beta-plane Geophysical Research Letters, 9, 769-772.

Boyd, J. P. and Z. D. Christidis, 1983: Instability on the equatorial beta-plane In J. Nihoul (Ed.), Hydrodynamics of the Equatorial Ocean (pp. 339-351). Amsterdam: Elsevier.

Boyd, J. P. and Z. D. Christidis, 1987: The continuous spectrum of equatorial Rossby waves in a shear flow Dynamics of Atmospheres and Oceans, 11, 139-151.

Boyd, J. P. and A. Natarov, 1998: A Sturm-Liouville eigenproblem of the Fourth Kind: A critical latitude with equatorial trapping Stud. Appl. Math., 101, 433-455.

Dickinson, R. E., 1968: Planetary Rossby waves propagating vertically through weak westerly wind wave guides Journal of the Atmospheric Sciences, 25, 984-1002.

Dunkerton, T. J., 1983: A nonsymmetric equatorial inertial instability $J$. Atmos. Sci., 40, 807-813.

Gill, A. E., 1982: Atmosphere-Ocean Dynamics, volume 30 of International Geophysics. Boston: Academic.

Hitchman, M. H., C. B. Leovy, J. C. Gille, and P. L. Bailey, 1987: Quasistationary zonally asymmetric circulations in the equatorial lower mesosphere J. Atmos. Sci., 44(16), 2219-2236.

Jones, D. S., 1997: Introduction to Asymptotics: a Treatment Using Nonstandard Analysis. Singapore: World Scientific 160 pp.

Lindzen, R. S., 1990: Dynamics in Atmospheric Physics. Cambridge: Cambridge University Press.

Marinone, S. G. and P. Ripa, 1984: Energetics of the instability of a depth independent equatorial jet Geophys. Astrophys. Fluid Dyn., 30(1-2), 105130. 
Moore, D. W. and S. G. H. Philander, 1977: Modelling of the tropical oceanic circulation In E. D. Goldberg (Ed.), The Sea, number 6 in The Sea (pp. 319-361). New York: Wiley.

Paris, R. B. and A. D. Wood, 1995: Stokes phenomenon demystified IMA Bulletin, 31, 21-28.

Philander, S. G. H., 1990: El Niño, La Niña and the Southern Oscillation, volume 46 of International Geophysics Series. Boston: Academic Press.

Proehl, J. A., 1996: Linear stability of equatorial zonal flows J. Phys. Oceangr., 26(4), 601-621.

Proehl, J. A., 1998: The role of meridional flow asymmetry in the dynamics of tropical instability J. Geophys. Res., 103(C11), 24597-24618.

Ripa, P., 1983: General stability conditions for zonal flows in a one-layer model. on the beta-plane or sphere Journal of Fluid Mechanics, 126, 463-489.

Ripa, P., 1991: General stability conditions for a multilayer model J. Fluid Mech., 222, 119-137.

Ripa, P. and S. G. Marinone, 1983: The effect of zonal currents on equatorial waves In J. C. J. Nihoul (Ed.), Hydrodynamics of the Equatorial Ocean (pp. 291-317). Amsterdam: Elsevier.

Segur, H., Tanveer, S., \& Levine, H., Eds., 1991: Asymptotics Beyond All Orders. New York: Plenum 389pp.

Stevens, D. E., 1983: On symmetric stability and instability of zonal mean flows near the equator J. Atmos. Sci., 40, 882-893. 